\title{
A Challenge to Diagnose Amalgam Associated Lichenoid Reaction in Elderly Patient
}

\author{
Anzany Tania Dwi Putri Baringbing ${ }^{1}$, Indriasti Indah Wardhany ${ }^{2 *}$ \\ 1. Oral Medicine Residency Program, Faculty of Dentistry, Universitas Indonesia, Jakarta 10430, Indonesia \\ 2. Oral Medicine Department, Faculty of Dentistry, Universitas Indonesia, Jakarta 10430, Indonesia
}

*E-mail: indriasti.indah61@ui.ac.id

\begin{abstract}
Introduction: Several studies reported the association between erosive oral lichenoid reaction (OLR) and amalgam which often arises without symptoms and rarely becomes chief complaint of patient in contrast with its local inflammatory reaction. Amalgam filling was popular in elderly patients. A decline in the protective functions of the oral mucosa could expose elder people to a variety of chemicals and put them in the higher risk to suffer from OLR.
\end{abstract}

Case Report: A 70-year-old female patient has chief complaints of taste loss and dry mouth. In the next scheduled visit, operator found a non-scrapable reticular with a reddish inflamed area on buccal mucosa adjacent to amalgam fillings which was painful when being stretched. The Amalgam filling associated OLR diagnosis was established. The lesion was reduced significantly after replacing amalgam filling followed by topical application of steroid. Many factors may contribute as the challenging factors for undetected OLR lesion during the first visit such as elderly altering mental status and pain perception.

Conclusion: Although the Amalgam filling associated OLR was a relatively common lesion in the elderly, factors that contribute as challenging factors to diagnose OLR must be comprehended by the dentist, in order to have a precise diagnosis.

Keywords: amalgam filling, elderly patient, oral lichenoid reaction

\section{Introduction}

The number and proportion of elderly people were steadily increasing in most countries worldwide. Among the population nowadays, approximately 600 million were aged 60 or older. As the consequences of this changing, the oral health patterns have also changed. ${ }^{1}$ In addition to dental caries and periodontal disease, oral mucosal disease is another significant problem found in elderly populations. $^{2}$

The oral mucosa has many essential protective functions that significantly affect the general health of an individual. With age, the oral mucosa becomes more vulnerable to many toxic substances and external carcinogens due to the alteration of its internal structures, such as the oral epithelium become thinner and collagen synthesis by connective tissue decreases. A decline in the protective functions of the oral mucosa could expose elder people to a variety of pathogens and chemicals that enter the oral cavity. $^{2}$

Besides that, elderly people may be related to systemic condition, such as metabolic changes, nutritional factors, or failures in organ functions that continuously consume multiple drugs every day. 3 Nevertheless, many adverse effects of these drugs have been reported arisen commonly among them, resulting in drug-associated oral diseases/condition, e.g. xerostomia, lichenoid reactions, or gingival hyperplasia. ${ }^{4}$ On the other hand, the mental status and pain perception in elderly people may also be altered. Anxiety may affect twice as many older adults as depression and it may be the most common mental alteration among them, especially when the complaints come with pain. But if the disease does not, they rarely notice and only focus on what causes discomfort.

In the dental practices, substances were identified as allergenic include local anesthetics, antibiotics, restorative materials, and latex. Silver amalgam has been used as a dental restorative material for over years and still remains the most commonly placed filling material in the world. ${ }^{5}$ Most elderly patients choose amalgam as their filling material because it is easily placed, relatively cheap, and durable. ${ }^{6}$ During the last decades, several studies reported the possible association between oral lichenoid reaction (OLR), regarded as either a clinical manifestation of a delayed hypersensitivity reaction or a chronic non-specific toxic reaction, and amalgam filling ${ }^{7}$, especially its local inflammatory reaction due to its toxic, irritants, or allergic effect. The biodegradation of this material will be 
decreasing its biocompatibility so the release of elements is nearly always related to adverse reaction arisen ${ }^{8}$.

The OLR is a lesion clinically and histologically indistinguishable from the oral lichen planus, but OLR will disappear when the causing substance is eliminated.5 Amalgam associated OLR often arises without symptoms so it rarely becomes a chief complaint of patient 9 whereas its most important complication is the possible malignant transformation.10 Thus, by altered mental status, pain perception, and mucosa structure and flexibility, and also the presence of other complaints arising pain and discomfort in elderly people, it will be a challenge for the dental professionals to diagnose oral lichenoid reaction at the early stage.

\section{Case Report}

A 70-year-old female patient came to Department of Oral Medicine, referred by Department of Periodontology, with complaints of taste loss in two third anterior of tongue since 6 months ago and dry mouth especially at night. She had hypertension and a history of Bell's Palsy of left region in 2013 so she consumed multiple drugs, such as Amlodipine, Aspilet, Mecobalamin, and Gabapentin routinely. She liked to suck lips, mouth-breathed, and snored while sleeping.

Examination revealed the dryness of lips and pallor of sclera. The patient's oral hygiene was fairly good. Only upper anterior gingiva was dry. Consistency of saliva on the floor of mouth was bubble. There was yellowish coating in two third posterior of tongue and a painless white lesion adjacent to the amalgam fillings on teeth no. 44 in right buccal mucosa.
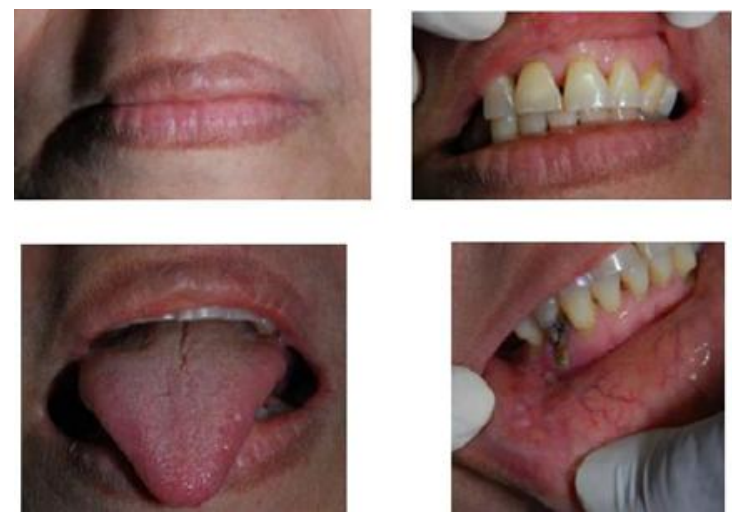

Figure 1. Oral Condition of the patient in first visit

The patient was informed that her complaints might be related to systemic condition and multiple drugs she had consumed. She was suggested to rinse her mouth with salt mixed water 3 times a day, use Vaseline to her dry lips, and brush her tongue gently twice a day.

Two weeks after that, patient returned to clinic. She felt progression to taste food but her mouth was drier after rinsing with salt mixed water. A blood test was done and the result was normal. The lesion on the right buccal mucosa became erosive and painful and it looked more clearly when the mucosa was being stretched. It showed a reticular pattern with a reddish inflamed area surrounding it. The lesion was nonscrapable and approximately $10 \mathrm{~mm}$ in length extending from first premolar to second premolar.
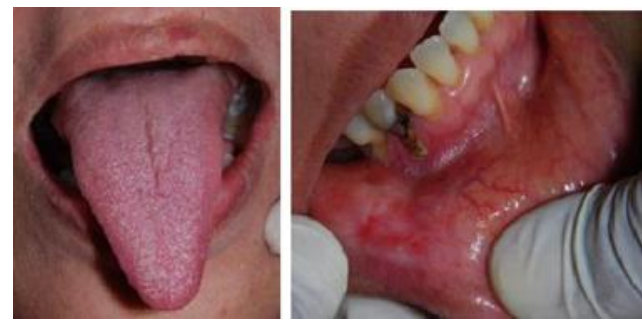

Figure 2. OLR became more erosive in second visit

A working diagnosis for this lesion was Oral Lichenoid Reaction (OLR) that was associated with fractured amalgam restoration. The patient was suggested to replace the amalgam restoration with a nonmetallic filling, be given $0.1 \%$ triamcinolone acetonide 3 times a day, and stop rinsing with salt mixed water. Follow up was advised.

Seven days later, she came to follow up. The dryness of mouth was less; only of upper anterior gingiva was still sustained. The replacement of amalgam restoration was done using glass ionomer cement (GIC) but there was a small crack in servical area, so she was suggested to repair her restoration to prevent teeth hypersensitivity. For the dryness of upper anterior gingiva, patient was informed that it might be related to her mouth-breathing and snoring at night. She was suggested to stop her bad habits, use GC Dry Mouth every bed time, and continue $0.1 \%$ triamcinolone acetonide for 7 days. Follow up was still advised.
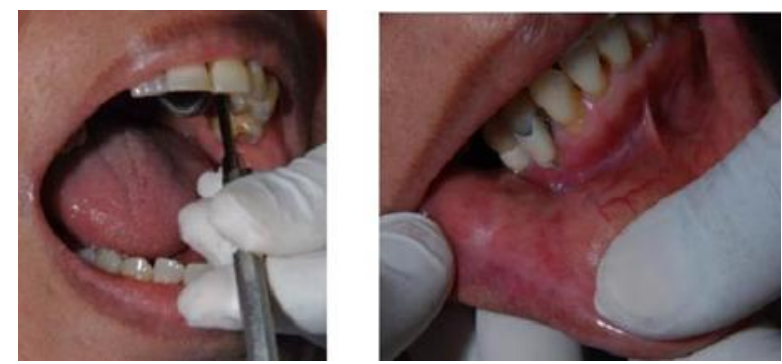

Figure 3. The appearance of OLR in third visit

Two weeks later, she returned to control. She had repaired her GIC filling on teeth no. 44. The dry mouth was decreased after using GC dry mouth 1-2 times every night and Vaseline 3 times a day. The $0.1 \%$ Triamcinolone acetonide was used only once a day but the OLR was reduced in size and severity. Patient was also suggested to consume fruits containing vitamin A more often and come to follow up her lesion once a month. 

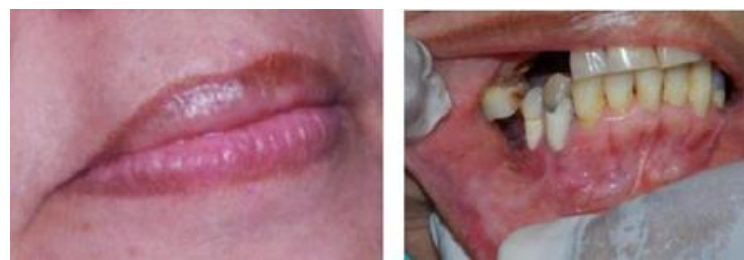

Figure 4. The OLR was healed in 4th visit

\section{Discussion}

The term "oral lichenoid reaction" (OLR) is commonly used to describe oral lichen planus (OLP) lesions that develop in contact with dental restoration, associated with consuming medications, graft versus host disease, or systemic conditions. The rate of prevalence of OLR is higher in women compared with men at the age group of 45-65 years. ${ }^{5}$ Clinically, these lesions cannot be distinguished from OLP. The morphology features included white or red patches, erythema, erosions, and ulcerations, with or without pain. ${ }^{8}$

In elderly patient, the OLR is often quite difficult to diagnose. Other than the fact that it may be asymptomatic and rarely become a chief complaint, the changes of mucosa structure and the presence of other complaint will harden to diagnose earlier. It is common for elderly people to focus on complaints that significantly arise pain and discomfort. In this patient, at first visit, she was disturbed with her loss of taste and dry mouth that made her disable to eat well. The clinical appearances of OLR had just been noticed in second visit when mucosa was being stretched wider. It might be caused by the alteration of mucosal flexibility and pain perception. This perception may alter due to the increase of pain threshold. It usually happens when the disease has occurred chronically. As a result, the lesion of OLR in this patient had lately found and already been more erosive. Furthermore, if the OLR has transformed to become erosive, it is reported to possibly transform to be malignant. ${ }^{10,11}$. All these conditions actually can be prevented if only the causative factor is removed earlier.

This case report also supports the growing evidences of amalgam restoration as etiologic factor in some of the mucosal changes. Side effects from a dental restorative material can be either irritative or allergic in nature that may represent the oral manifestation in some patients or be the clinical result of a delayed hypersensitivity reaction in others. A chronic toxic reaction may be established due to repeated or constant zone with the toxic agent. Chronic toxic reactions may be seen in areas of the oral mucosa in direct contact with restorations. Allergy to filling compounds may be able to consider because the remission of this lesion is shown after the replacement of amalgam. ${ }^{8,11}$

Most elderly people also commonly consume multiple drugs routinely related to their systemic condition that some can interfere sensory nerves in dorsum of tongue that may cause loss of taste8, but the drugs that she consumed are not included on the drugs list that could induce OLR lesion.12 Another cause of OLR may be immunological or toxic reactions to plaque accumulation. Such lesion may disappear after improved oral hygiene. Plaque reduction may also have surprising effects on mucosal lesions.

In this patient, the food tastes progressed better after she brushed her teeth properly, cleansed her tongue, and swished with salt-mixed water twice a day. For the OLR, elimination of causative factor needs to be done. The replacement of amalgam into nonmetallic filling, such as GIC, gave reduction in size and severity for the lesion. The using of $0,1 \%$ triamcinolone acetonide as a topical steroid also accelerated the healing process. But unfortunately, most elderly patients have compliance burden, including this patient. It might be related to the remission of memory capacity. Thus, support from family's member is needed to achieve the goal of this treatment.

\section{Conclusions}

From this case report, it is evident that amalgam restorations may induce oral lichenoid reaction (OLR) in elderly patient but this lesion is rarely symptomatic and not a chief complaint. It may result that many general practitioners are unaware of their existences. The replacement of amalgam into nonmetallic filling and using $0.1 \%$ triamcinolone acetonide gently to the lesion may improve the progression of OLR, but follow up once a month is advised at least a minimum of 3 months ahead.

\section{References}

1. Ingela Grönbeck-Linden1, 2, Catharina Hägglin1, 2 AP, Per O. Linander1 LG. Discontinued dental attendance among elderly people in Sweden. J Int SocPrev Community Dent; 2016.

2. Sadeq Ali Al Maweri, Aisha Ahmed Al Jamaei, Ghadah A. Al Sufyani, Bassel Tarakji BS-A. Oral mucosal lesions in elderly dental patients in Sana'a, Yemen. J Int Soc Prev Community Dent; 2015.

3. Cueto A, Mart??nez R, Niklander S, Deichler J, Barraza A, Esguep A. Prevalence of oral mucosal lesions in an elderly population in the city of Valparaiso, Chile. Gerodontology. 2013;30(3):201-doi:10.1111/j.1741-2358.2012.00663.x.

4. Anil Govindrao Ghom SA (Lodam) G. Section 5: Misscellanous. In: Textbook of Oral Medicine. ; 2014:1032.

5. Pawar R, Mattigatti S, Mahaparale R, Kamble A. Lichenoid reaction associated with silver amalgam restoration in a Bombay blood group patient: A case report. J Conserv Dent. 2016;19(3):289. doi:10.4103/0972-0707.181950.

6. Wilson J. Amalgam as a filling material for the older person - A personal opinion. Gerodontology. 2014;31(4):241-242. doi:10.1111/ger.12165.

7. Henriksson E, Mattsson U, Hakansson J. Healing of lichenoid reactions following removal of amalgam. A clinical follow-up. J Clin Periodontol. 1995;22(4):287- 294. Available http://www.ncbi.nlm.nih.gov/entrez/query.fcgi?cmd=R etrieve \&db=PubMed\&dopt=Citation\&list_uids $=76226$

8. Issa Y, Duxbury a J, Macfarlane T V, Brunton P a. Oral lichenoid lesions related to dental restorative materials. $\mathrm{Br}$ Dent J. 2005;198(6):361-366; disussion 549; quiz 372. doi:10.1016/S0084-3717(08)70166-1.

9. Sirkku Niissalo, Goran Hampf, Jarkko Hietanen, Maria Maimstrom, Svetlana Solovleva, Antti Pertovaara YTK. 
Thermal Sensation and Pain in Oral Lichen Planus and Lichenoid Reaction. Oral Pathol Med; 2002.

10. Taghavi Zenouz a, Mehdipour M, Attaran R, Bahramian a, Emamverdi Zadeh P. Squamous cell carcinoma arising from an oral lichenoid lesion: a case report. J Dent Res Dent Clin Dent Prospect. 2012;6(1):29-32. doi:10.5681/joddd.2012.007.

11. Lind PO, Hurlen B, Lyberg T, Aas E. Amalgam-related oral lichenoid reaction. Scand J Dent Res. 1986;94(5):448-451.

12. P Serrano-Sánchez 1, JV Bagán 2, Jiménez-Soriano 3 GS Drug-induced oral lichenoid reactions. A literature review. J Clin Exp Dent. 2010;2 [cited 2010 April]:e71-e75. doi:10.4317/jced.2.e. 